\title{
marges Marges
}

revue d'art contemporain Revue d'art contemporain

$08 \mid 2008$

L'art à l'heure de la société de services

\section{Web 2.0 : veuillez créer s'il vous plaît}

Web 2.0: Would You Mind Creating Please

Jean-Noël Lafargue

\section{(2) OpenEdition}

Journals

Édition électronique

URL : http://journals.openedition.org/marges/578

DOI : $10.4000 /$ marges. 578

ISSN : 2416-8742

\section{Éditeur}

Presses universitaires de Vincennes

\section{Édition imprimée}

Date de publication : 15 octobre 2008

Pagination : 102-109

ISBN : 978-2-84292-252-8

ISSN : $1767-7114$

Référence électronique

Jean-Noël Lafargue, «Web 2.0 : veuillez créer s'il vous plaît », Marges [En ligne], 08 | 2008, mis en ligne le 15 octobre 2009, consulté le 01 mai 2019. URL : http://journals.openedition.org/marges/578 ; DOI : $10.4000 /$ marges. 578 


\section{Web 2.0: veuillez créer s'il vous plaît}

«Web 2.0 » est le nom que l'on donne depuis quelques années à ce que la presse - à la suite des prospectivistes en nouvelles technologies - présente comme une évolution majeure d'Internet et dont on pourrait résumer le principe à une abolition technique et philosophique de la frontière qui distingue le public des sites Internet des auteurs du contenu de ces sites. La réalité est sans doute nettement moins enthousiasmante.

Techniquement, le Web 2.0 est un ensemble informel d'outils et de pratiques destinés à rendre les sites Internet plus immédiatement ouverts à des contributeurs qui ne maîtrisent aucune technologie de création de pages Internet. Le fait de pouvoir ajouter des commentaires aux articles du quotidien Libération ou aux fiches-produits d'Amazon est Web 2.0, le fait d'ouvrir son MySpace, son Facebook ou son FlickR est Web 2.o. Les blogs et les wikis sont eux aussi Web 2.o. Ces systèmes sont différents à de nombreux égards. Leur point commun est la facilité d'accès pour des non-spécialistes et, bien souvent, la constitution plus ou moins structurée de communautés d'utilisateurs.

Les activités proposées aux usagers du Web 2.0 consistent donc à produire des textes, des images, des séquences d'animation ou de musique (tout ce que le droit nomme « les œuvres de l'esprit »), de dialoguer, d'échanger ses impressions sur des produits de consommation ou sur des spectacles, mais aussi parfois d'attribuer des notes aux autres contributeurs ou à leurs travaux. Quelques uns de ces sites ont leurs " employés du mois », à la manière de ce qui se fait dans certaines entreprises, et récompensent (parfois sous forme de gratification symbolique) les contributeurs dont les productions auront été jugées comme étant les plus intéressantes et les plus prolifiques. Par exemple, Ciao.fr ("la communauté shopping », site d'origine allemande décliné en de nombreuses langues) propose à chacun de rédiger un article sur sa brosse-à-dents, son après-rasage ou sur un yaourt. Les auteurs de critiques sont rémunérés lorsque d'autres inscrits attribuent des notes favorables à leurs textes. Les rémunérations sont extrêmement mesquines. Pour 
les compléter, les rédacteurs peuvent répondre à des sondages rémunérés. L'usager du site se trouve donc servir un peu à tout: rédacteur, membre du comité de lecture, censeur/délateur, mais aussi cible d'études commerciales et même de messages publicitaires puisque ce " portail de la consommation » sert principalement de support à des réclames. Entreprise prospère, Ciao a été rachetée récemment pour plus de 150 millions de dollars par Greenfield, une société d'études commerciales.

En observant ce système, on constate que les textes fournis par les rédacteurs quasi-bénévoles du site manquent cruellement d'intérêt: vite et mal écrits (on peut estimer la valeur financière du feuillet à quelques centimes d'euro, ce qui impose un rendement soutenu à ceux qui comptent s'enrichir de cette façon), ils apportent rarement des informations inédites à leurs lecteurs car leur contenu ne fait souvent que paraphraser l'emballage et les argumentaires commerciaux des produits chroniqués. En effet, si des rédacteurs sont attirés par ce genre de site pour assouvir leur besoin de parole ou leur graphomanie, le système qu'on leur impose est suffisamment contraignant pour les amener à standardiser, à aplatir leur prose. Un certain ton, un certain débit (les notes pénalisantes sont souvent accompagnées des remarques « trop long » ou « trop court ») et des échanges de bons procédés entre contributeurs sont l'assurance de recevoir les "bonnes notes » qui entraînent rémunération. En revanche, écrire sur des sujets autres que des produits de consommation (une ville, par exemple) ou sur des produits culturels (film, livre) n'entraîne aucune rémunération - sans doute parce que ces sujets sont ceux qui attirent le plus de rédacteurs bénévoles: nul besoin de payer. Les propriétaires du site se réservent par ailleurs le droit absolu de supprimer tout article qui ne leur plairait pas pour une raison ou une autre.

L'exemple de Ciao.fr est caricatural, il détourne le besoin de communiquer pour faire du consommateur le lecteur actif des publicités dont il est la cible et le co-auteur en contrepartie d'une gratification financière qui s'avère symbolique.

Le volume de pages présentes sur un tel site, impossible à obtenir autrement qu'avec le concours de bénévoles, lui permet d'avoir un Google rank ${ }^{1}$ exceptionnel. La qualité des commentaires sur les produits n'a donc aucun intérêt informatif spécifique - non hiérarchisés, rédigés sans méthodologie, généralement contradictoires mais leur quantité offre au site et aux produits qu'il promeut une visibilité importante.

De nombreux sites de vente par correspondance permettent à leurs usagers de noter et de commenter les produits qui figurent à leur
${ }^{1}$ Le Google rank est la position à laquelle apparaît un site dans le résultat d'une recherche effectuée avec Google. 
catalogue: Fnac.com, Amazon, Pixmania, Ldlc, Boursorama, etc. Presque toujours, les commentaires peuvent eux-mêmes être évalués et contrôlés par d'autres utilisateurs qui se chargent de dénoncer toute prose inappropriée. L'ordre d'affichage des textes est souvent indexé sur la satisfaction exprimée vis-à-vis du produit, du plus enthousiaste au moins enthousiaste. Les rédacteurs qui attribuent systématiquement des notes de cinq étoiles voient donc leurs écrits nettement mieux mis en valeur que ceux d'auteurs plus sévères. Un tel fonctionnement permet aux sites marchands de se décharger d'une grande partie de leur travail sur leurs propres clients. De manière tout aussi rusée, ces services proposent à leurs usagers d'être leurs principaux agents de promotion grâce à une fonction de type " signalez ce produit à un ami » (qui aboutit à l'envoi d'un email) : la responsabilité de la publicité est transférée, elle devient l'affaire de particuliers. Il est en effet irritant de recevoir une publicité non-sollicitée mais, émanant d'un ami, cette publicité devient une recommandation et, si l'on tient à se fâcher, ce sera contre l'ami. Tous les sites Web 2.0 commerciaux proposent un contrat à leurs utilisateurs. Un contrat qui n'est pas toujours lu jusqu'au bout et qui contient, pour l'essentiel, trois clauses importantes. La première clause, c'est le dégagement de responsabilité. En cas de procédure judiciaire quelconque, c'est l'auteur des textes ou des images qui est pénalement responsable. Pour cette raison, la quasi-totalité de ces sites demandent leurs coordonnées véritables à ceux qui y contribuent. La seconde clause, c'est l'absence de garanties que fournit le service. Si le serveur qui abrite l'ensemble des photographies (FlickR par exemple) ou les travaux bureautiques (Google document) d'un usager venait à tomber en panne, ce serait le problème du dit usager. Et si du jour au lendemain, Skyblog, Blogspot, Youtube, DeviantArt ou autres décidaient qu'un contenu leur déplaît pour une raison quelconque, le contrat passé avec l'utilisateur permet à ces services de supprimer purement et simplement le contenu en question. Certains suppriment d'ailleurs les blogs trop peu souvent mis à jour - ils encombrent mais ne rapportent pas suffisamment de recettes publicitaires puisque le succès d'un blog dépend notamment de la régularité de son actualisation. La troisième clause, enfin, qui justifie parfois la seconde, c'est le renoncement des usagers du site à leurs droits patrimoniaux. Sur ce point, tous les services n'ont pas la même politique. Certains exigent de leurs contributeurs qu'ils renoncent intégralement à la paternité de leurs œuvres, d'autres, de plus en plus souvent (car même s'il n'y a pas de jurisprudence à ce sujet, on peut supposer que de tels contrats seraient jugés léonins par la justice), se contentent de définir 
le cadre de diffusion des œuvres pour protéger leurs arrières, financièrement parlant: aucun droit d'auteur ne saurait être réclamé.

Le point commun de ces services Web 2.0 est donc effectivement l'abolition des distinctions entre le public d'un site Internet et ses contributeurs, mais cela se fait plutôt au détriment des usagers qu'à celui des gestionnaires de ces systèmes. Aux premiers le labeur et les risques; aux seconds les bénéfices qui en découlent. La transaction est suffisamment déséquilibrée pour que nous devinions le paramètre qui la rend possible: la loi de l'offre et de la demande. Il y a sans doute beaucoup plus de gens qui ont envie d'être lus, de montrer leur production visuelle ou musicale, d'exister en somme, que de gens qui sont en mesure d'exiger une contrepartie financière à l'exposition de leurs productions.

Au-delà des sites destinés à accueillir les reviews [critiques] et autres " réactions ", certains services entendent offrir un véritable espace créatif à leurs usagers. C'est le cas des blogs (web logs) notamment, très populaires. Il en existe deux sortes bien distinctes: ceux qui sont gérés par leurs rédacteurs et ceux qui sont gérés par une société (Canalblog, Skyblog, Blogspot, etc.). Les premiers sont des logiciels, souvent gratuits, que leurs utilisateurs doivent installer eux-mêmes sur des serveurs. Wordpress et Dotclear sont les deux plus célèbres. Hautement configurables, ils peuvent être difficiles à installer puis à maîtriser techniquement, ne serait-ce que parce qu'ils nécessitent de comprendre ce qu'est un serveur et comment on y télécharge des fichiers. C'est cette difficulté relative qui fait le succès du second type, celui des blogs gérés par des sociétés. Avec Canalblog, par exemple, on peut créer son journal en quelques minutes - en trois « clics », nous dit-on - et sans frais. L'intérêt des sociétés qui gèrent les blogs est financier, car le contenu visuel ou textuel que fournit gracieusement l'utilisateur sert de support à des publicités souvent ciblées (se rapportant au contenu des textes du blog). En théorie, l'expression est libre sur ces blogs. Bien entendu, les contenus illégaux (diffamation, menaces, incitation à commettre des crimes, contravention au droit d'auteur) sont proscrits. La plupart des blogs, à l'exception de ceux qui en font une spécialité, interdisent aussi la pornographie. Mais ce qu'aucun ne manque d'interdire à ses usagers, c'est la diffusion de publicités autres que celles qu'impose, à son profit exclusif, la société gestionnaire des blogs. Pour donner un exemple, le service Over-blog, qui est sans doute le plus respectueux des auteurs (qui conservent leur droit d'auteur et perçoivent même une part de la rémunération issue de la publicité), rédige ses conditions générales en respectant les règles élémentaires de la typographie mais emploie abruptement 
2 Étienne Mineur, «Peut-on encore être graphiste au pays des Templates?", mercredi 8 août 2007 (http://www.my-os.net). les capitales pour avertir: "LA PUBLICITÉ SANS ACCORD DE LA PART DE LA SOCIÉTÉ JFG NETWORKS EST INTERDITE SUR UN BLOG UTILISANT LE SERVICE OVER-BLOG ».

Les conditions d'utilisation du service Canalblog établissent quant à elles une définition assez savoureuse du mot blog, qui serait, nous dit-on, une « page Web évolutive et non conformiste présentant des informations de toutes sortes, généralement sous forme de courts messages mis à jour régulièrement [...]». On peut s'interroger sur l'usage un brin cynique de la locution " non conformiste » lorsqu'elle est employée pour décrire un système de publication qui standardise les contenus et leur organisation et transforme le principe du journal intime en un véritable mass-media.

Dans un article publié sur son blog, «Peut-on encore être graphiste au pays des Templates²? ", Étienne Mineur s'interroge sur le rôle dans lequel les interfaces standardisées (blogs et autres) placent les créateurs de sites Internet. Du rôle de concepteurs d'interfaces qu'ils avaient parfois aux débuts du web et à l'époque du CD-Rom, les designers graphiques sont à présent relégués au rôle d' "habilleurs» de sites, de "customiseurs» interchangeables, puisque les différentes couches (ergonomie, système d'affichage, graphisme et contenu) sont désormais séparées: "Avec les nouveaux sites web et autres applications interactives, le designer/graphiste perd son contrôle total sur la forme de l'objet. Il doit jouer avec des possibilités, des contraintes, des extrêmes... il donne des indications, il dirige graphiquement plutôt qu'il ne maîtrise totalement la forme du produit son travail. ». Pour Étienne Mineur, il n'existe plus qu'une alternative pour les designers graphiques: abandonner Internet ou, à l'opposé, assimiler toutes les nouveautés techniques et savoir les utiliser.

Contrairement à ce qu'affirment les thuriféraires du Web 2.0, l'histoire du World Wide Web ne tend peut-être pas à donner à chacun les moyens de s'exprimer, mais au contraire - c'est la thèse que nous retenons en tout cas -, n'est qu'une succession de paliers vers une prolétarisation des créateurs de sites web, prolétarisation au sens où ils sont dépossédés de leurs moyens de production.

Le premier web, disons le web zéro, créé par des ingénieurs du CERN en 1991, servait à rédiger des documents scientifiques de manière structurée. Les possibilités de mise en forme des premières pages web étaient pour le moins rudimentaires : listes, paragraphes, gras, italiques, du texte noir sur fond gris. La grande idée n'était pas visuelle, mais fonctionnelle: ces pages pouvaient contenir des liens vers d'autres pages (liens qui s'affichaient en bleu vif). Deux ans plus tard, le CERN autorise le monde entier à utiliser le 
protocole « http:// » - la navigation sur Internet - et le langage HTML (un langage de description de pages, lui-même dérivé d'un langage plus complexe, le SGML). C'est la naissance, si l'on veut, du «Web 1 ». De nouveaux logiciels de navigation, comme le très populaire Netscape, sont diffusés et imposent peu à peu de nouveaux standards au web, répondant à une demande du public qui veut de la couleur, un choix typographique, des images, une interactivité plus évoluée et même, des séquences animées et sonores. En effet, le web ne sert plus seulement à relier des documents scientifiques mais s'ouvre à toutes sortes de pratiques, à commencer par les homepages que des passionnés consacrent à leurs marottes. Le site Internet Movie Database qui n'était au départ qu'une liste de films établie par un simple amateur est ainsi devenu, quinze ans plus tard, un acteur incontournable dans l'industrie du cinéma. Viendront ensuite les sites corporate [d'entreprise] qui permettent à des sociétés ou des organismes divers d'expliquer leurs produits ou leurs actions. Et avec ces sites, la création sur Internet passe de l'amateurisme au professionnalisme. Les métiers de webmaster ou web-designer se popularisent. Les personnes employées à ces tâches sont souvent des autodidactes échoués là par le hasard ou la nécessité. En France, vers la fin des années 1990, des usines à sites Internet tels que La Mine ou le Studio Grolier recrutent plus volontiers des auteurs de pages personnelles à succès que des informaticiens diplômés. Ces derniers sont eux affectés à des tâches "lourdes » telles que l'administration des réseaux et des serveurs ou le développement d'applications très spécifiques tâches techniques mais souvent créatives et enthousiasmantes. Cette époque est aussi celle des premiers hébergements web associatifs ou philanthropiques pour pages personnelles d'amateurs: Mygale.org ${ }^{3}$ et Altern.org notamment. Le premier, en pleine crise de croissance, acceptera de frayer avec les start-ups et sera rapidement digéré par Multimania/La Baguette Virtuelle puis par Lycos. Le second, d'inspiration nettement libertaire mais appartenant à une société, sera étranglé par des procès (contrefaçon, diffamation, atteinte au droit à l'image, racisme) relatifs au contenu des sites hébergés (Caliméro, RATP, Estelle Halliday, Jean-Louis Costes), puisqu'à l'époque, la justice assimile les hébergeurs de sites Internet à des éditeurs.

Du fait même de ses origines, le web se révèle toujours trop limité. Lassés de l'astuce et du bricolage, les webdesigners amateurs ou professionnels accueillent donc avec enthousiasme les évolutions successives des outils de création de sites: le langage Javascript qui rend la page web active; le langage Css qui autorise l'application de
${ }^{3}$ Au départ un projet universitaire de Frédéric Cicera, chercheur en Intelligence Artificielle à l’Université Paris 8. 
${ }^{4}$ Mentionnons l'artiste André Lozano, auteur du système de publication Loz (http://logz.org/). styles graphiques rationalisés; les langages Perl, Php, Java, Ruby, $\mathrm{Xml}$, qui permettent la communication avec des bases de données et donc à un contenu dynamique; les plug-ins Quicktime, Flash et Shockwave, qui permettent l'utilisation d'animations évoluées et l'inclusion de séquences sonores ou animées, etc.

Dans un but de performance, beaucoup de ces outils sont devenus aujourd'hui extrêmement stricts et difficiles à manier; ce qui a deux conséquences principales. La première, c'est qu'ils découragent les amateurs, ces derniers se tournent donc vers des systèmes qui occultent les questions techniques - le fameux Web 2.0. La seconde conséquence, c'est que ces outils définissent fortement ce que doit être Internet et la manière dont son contenu doit s'organiser. Les pratiques se standardisent et dans une certaine mesure, les contenus aussi. Par exemple, choisir le système des blogs impose presque obligatoirement de présenter ses articles sous forme de billets organisés chronologiquement, ce qui ne saurait être adapté à toutes les sortes de contenus.

Tout ceci aboutit à distinguer autoritairement les métiers, les moyens et donc les personnes. L'évolution technique qui n'est pas un artifice (le système du blog, par exemple, est véritablement pratique pour celui qui y a recours) est donc responsable, osons le mot, d'une taylorisation du web personnel. Comme la taylorisation spécialise l'ouvrier et le prive de la visibilité du processus dans lequel s'inscrit son travail, la division des tâches sur Internet profite avant tout à ceux qui l'organisent. Une telle évolution, de l'amateurisme à l'artisanat puis de l'artisanat à l'industrie, constitue sans doute un processus banal, mais nous avons en tout cas pu assister à ce tournant de la création sur Internet. Il est plus étonnant que cette évolution touche autant, sinon plus, les sites Internet amateurs.

Pour qu'une création numérique véritable et libre continue à exister, il semble important que les créateurs, notamment les artistes, soient conscients des implications de leurs choix techniques et ne transfèrent pas ingénument le contrôle de leur propre production à des sociétés dont les objectifs ne peuvent correspondre aux leurs que sur un malentendu. Plusieurs approches nous semblent possibles: - Conserver la maîtrise des aspects techniques de la publication web pour n'être dépendant de personne, être le propriétaire et même le constructeur de ses propres outils de création et de diffusion$^{4}$. C'est l'approche, y compris hors-ligne, de John Maeda, qui étend la question à tout logiciel: pour lui, utiliser une application telle que Word, Photoshop, Illustrator ou encore Flash revient à accepter de contraindre sa production aux possibilités prévues par ceux qui ont mis au point ces outils. 
- N'utiliser que des outils qui appartiennent collectivement à leurs usagers, comme c'est le cas de Mediawiki, le moteur de l'encyclopédie Wikipédia. C'est le principe du « libre», que certains étendent aux œuvres elles-mêmes, allant même jusqu'à refuser toute captation exclusive des idées à commencer par les leurs 5 .

- Retourner les outils standardisés contre eux-mêmes, les détourner de leur usage naturel, les « hacker » ou au contraire les utiliser en poussant leur logique au maximum 6 . Ce type d'intervention permet au passage de découvrir les limites non explicitement écrites qui encadrent la créativité des usagers de ces services. L'artiste Christophe Bruno a par exemple constaté à ses dépens que le service Adsense de Google (qui permet en quelques secondes de lancer une campagne publicitaire sur Internet, que l'on soit un particulier ou non) accepte toutes sortes d'annonces publicitaires, mais refuse absolument les textes poétiques ou obscurs, enfin tout ce qui n'est pas à vendre?. D'autres ont découvert que l'on pouvait être banni de ce même service en en dévoilant publiquement le fonctionnement.

- La parodie est bien entendu une autre manière de résister à la standardisation, par l'observation et le recul ${ }^{8}$.

Il semble en tout cas difficile à ceux qui veulent utiliser Internet comme support ou comme sujet artistique de ne pas tenir compte de la question des limites à leur liberté de création que leur imposent ceux qui organisent le réseau et ses outils.

De manière pour le moins étonnante, il semble que beaucoup d'artistes ou de groupes d'artistes (囚TMark, Jodi, Vuk Cosic, eToy...) aient traité ces questions avant qu'elles ne deviennent véritablement urgentes, c'est-à-dire à une époque où les limites de la création sur Internet étaient surtout dues au fait que le réseau n'avait pas été créé pour répondre aux besoins des créateurs de contenus artistiques ou littéraires.

Si des questions doivent être posées, ce sont justement celles qui découlent du fait que le réseau encadre à présent les créateurs - au sens large - dont l'art est un cas particulier.
${ }^{5}$ Comme Antoine Moreau, promoteur de la licence " Art Libre " et de la création nonpropriétaire.

${ }^{6}$ Je citerai en exemple de nombreuses interventions de Claude Closky (http://www.sittes.net).

${ }^{7}$ Christophe Bruno, The Google AdWords Happening, 2002 (http://www.iterature.co m/adwords/).

${ }^{8}$ Citons par exemple Jean-Baptiste Bayle (http://myownspace.fr).

\section{Jean-Noël Lafargue}

